\title{
Radio-loud active galaxies in the northern ROSAT All-Sky Survey
}

\section{Radio identifications ${ }^{\star}$}

\author{
S.A. Laurent-Muehleisen ${ }^{1}$, R.I. Kollgaard ${ }^{1}$, P.J. Ryan ${ }^{1}$, E.D. Feigelson ${ }^{1}$, W. Brinkmann ${ }^{2}$, and \\ J. Siebert ${ }^{2}$ \\ 1 Department of Astronomy \& Astrophysics, The Pennsylvania State University, University Park, PA 16802, U.S.A. \\ 2 Max-Planck Institut für Extraterrestrische Physik, D-85740 Garching, Germany
}

Received March 13; accepted May 22, 1996

\begin{abstract}
We present $5 \mathrm{GHz}$ high resolution VLA observations of 2,127 radio- and X-ray-emitting sources found in both the Green Bank (GB) $5 \mathrm{GHz}$ radio catalog and the ROSAT All-Sky Survey (RASS). We report core flux densities and positions accurate to $\pm 0.5^{\prime \prime}$. Combined with the GB measurements of the total radio emission, we derive the core-to-lobe ratio of objects in our sample and discuss their core-dominance relative to samples of radio galaxies and BL Lacertae objects. Our results show the RASS/Green Bank (RGB) sample is approximately an order of magnitude more core-dominated than the radio galaxy sample, but is more than an order of magnitude less core-dominated than highly beamed BL Lacertae objects. Using simple beaming models, this indicates the typical object in the RGB catalog exhibits moderately beamed radio emission and is oriented at an angle to the lineof-sight $\bar{\theta}_{\mathrm{RGB}} \sim 25^{\circ}-35^{\circ}$. The case of the origin of the $\mathrm{X}$-ray emission is not as clear; the data are consistent with either an anisotropic unbeamed or moderately beamed $\mathrm{X}$-ray component. Tables 2 and 3 which present the RGB catalog are available in their entirety only from the CDS via anonymous ftp to cdsarc.u-strasbg.fr (130.79.128.5), via the WWW at http://cdsweb.ustrasbg.fr/Abstract.html, or at ftp://ftp.astro.psu.edu /pub/edf.
\end{abstract}

Key words: surveys - catalogs — radio continuum: galaxies - X-rays: galaxies - galaxies: active — quasars: general

Send offprint requests to: S.A. Laurent-Muehleisen

* Tables 2 and 3 only available in electronic form at CDS via ftp 130.79.128.5 or http://cdsweb.u.strasbg.fr/Abstract.html

\section{Introduction}

The catalog created from the ROSAT All-Sky Survey (RASS) consists of $\sim 60000$ sources making it the deepest complete sample of soft X-ray $(0.07-2.4 \mathrm{keV})$ sources ever constructed (Voges 1993). Previous X-ray surveys such as the HEAO-1 Large Area Sky Survey (Wood et al. 1984), the Einstein Extended Medium Sensitivity Survey (Gioia et al. 1990; Stocke et al. 1991), and the Einstein Slew Survey (Elvis et al. 1992), consisted of less than 5000 sources total. Identification programs of these earlier surveys showed the majority of objects are extragalactic consisting mainly of quasars, Seyferts, BL Lacertae objects, clusters of galaxies and occasionally normal galaxies. Similarly, the bulk properties of various samples of optically identified objects in the RASS (e.g. Brinkmann et al. 1994; Brinkmann et al. 1995; Bade et al. 1995) have shown that the RASS contains thousands of extragalactic objects and will therefore provide the largest flux-limited sample of X-ray-emitting AGN for the foreseeable future.

Because RASS positions are known to only $\sim 30^{\prime \prime}$ accuracy, complete identification of the entire RASS cata$\log$ is an enormous task. Correlations with deep surveys at other wavelengths can efficiently create subsamples of manageable size and also select objects of particular interest. We present here 2,127 sources which appear in both the RASS and the 1987 Green Bank (GB) 5 GHz radio survey (Gregory \& Condon 1991; Gregory et al. 1996, hereafter called GB96). Because $>70 \%$ of the sources in this RASS-Green Bank (RGB) sample are optically unidentified and the positional accuracy of both surveys is low, we obtained high resolution radio observations to enable the identification of unique optical counterparts. Out detection of compact core radio components of these radioloud active galaxies together with GB observations of the total radio emission, permits study of the beaming 
characteristics of these RASS sources. The multiwavelength properties of the previously optically identified sources appear in Brinkmann et al. (1995, B95) and multiband radio observations of a subset are given by Neumann et al. (1994).

This paper is organized as follows: the construction of the RGB catalog is discussed in Sect. 2 and the new radio data presented in Sect. 3. In Sect. 4 we compare our results to the Green Bank data. Section 5 uses simple beaming models to characterize the radio emission of the sources in our catalog and Sect. 6 discusses the X-ray beaming properties. The broadband multifrequency properties of the entire RGB catalog, including newly identified optical counterparts and X-ray properties, will be presented in Brinkmann et al. (1996; B96).

\section{The RASS-Green Bank (RGB) sample}

Analysis of the Green Bank radio data was performed by fitting single elliptical Gaussian surfaces to local enhancements (Neumann et al. 1994). The final catalog contains $\sim 150000$ small diameter sources above a detection threshold of $3 \sigma$. The flux density limit is $\sim 15 \mathrm{mJy}$ in the declination range from $30^{\circ}-75^{\circ}$ and increases to $\sim 24 \mathrm{mJy}$ at $0^{\circ}$ declination. In order to identify as many radio-emitting objects in the RASS as possible, this list was purposely constructed to be deeper than those lists published later (Becker et al. 1991; Gregory \& Condon 1991), which are restricted to $>5 \sigma$ sources. The initial radio source catalog is therefore likely to contain many faint spurious sources. The rate of spurious coincidences in the overall RASS-Green Bank correlation ought to be much less, however, since close proximity $\left(100^{\prime \prime}\right)$ to a detected X-ray source is required. The $1 \sigma$ positional accuracy of the Neumann et al. (1994) Green Bank catalog is approximately $\pm 15^{\prime \prime}$ for bright sources and $\pm 40^{\prime \prime}$ for fainter ones (Neumann et al. 1994). Direct comparison of the reanalyzed GB flux densities with those published in GB96 shows that for sources $>100$ mJy the flux densities are accurate to $\sim 20 \%$ while for sources $<100 \mathrm{mJy}$ the flux densities differ by up to $\sim 40 \%$, with the reanalyzed values generally being higher.

The RASS data were processed using the semiautomatic Standard Analysis Software System (SASS; Voges et al. 1992) which determines the sky coordinates and energy of each photon by applying an aspect solution and calibration to each X-ray event. The data are then analyzed using various source detection algorithms, comprising two sliding window techniques and a maximumlikelihood method. Further details are given in Voges (1993).

The RASS and GB surveys were cross-correlated producing a catalog of 2,127 sources with separations of $<100^{\prime \prime}$. These sources are listed in Tables $2-4$ below. The distribution of the separations is well represented by a Gaussian with $\sigma \sim 17^{\prime \prime}$ for distances up to $40^{\prime \prime}$ and is ap- proximately constant beyond this (Fig. 1, B95). Because of the highly non-Gaussian nature of the distribution, sources with RASS/GB separations of up to $100^{\prime \prime}$ were included in this initial catalog. The number of spurious coincidences is believed to be less than 200 objects (B95). The radio flux densities range from $15 \mathrm{mJy}$ to $60 \mathrm{Jy}$ and the $\mathrm{X}$-ray fluxes range from $810^{-14}$ to $410^{-10} \mathrm{erg} \mathrm{s}^{-1} \mathrm{~cm}^{-2}$.

\section{New VLA observations}

High resolution observations of the RGB sample were made with the NRAO's Very Large Array ${ }^{1}$ (VLA) between October 1992 and September 1995. The observations were recorded with the two standard $50 \mathrm{MHz}$ bandwidth IFs at an effective frequency of $4.885 \mathrm{GHz}$. Table 1 summarizes the observing parameters including the epoch, array configuration, average exposure time per source and beam size. On October 3, 1992, data were collected while the VLA was in a hybrid A/D configuration. We were able to obtain flux densities and positions for these sources only by using the antennas in the low-resolution D-like configuration which yielded insufficient positional accuracy for unambiguous optical identification. For completeness we list these sources separately but do not consider them part of our well-defined sample. They are excluded from further analysis. The region of the sky covered by the D-configuration observations is approximately defined by $0^{\mathrm{hr}}<\alpha<15^{\mathrm{hr}}, 0^{\circ}<\delta<40^{\circ}$ and $15^{\mathrm{hr}}<\alpha<16^{\mathrm{hr}}, 0^{\circ}<$ $\delta<15^{\circ}$, although high resolution observations for several objects in this region were obtained.

Table 1. Observing log

\begin{tabular}{lrlcccc}
\hline & Date & & $\begin{array}{c}\text { Obs } \\
\text { Code }\end{array}$ & $\begin{array}{c}\text { VLA } \\
\text { Config }\end{array}$ & $\begin{array}{c}\text { Exp. } \\
(\text { min })\end{array}$ & $\begin{array}{c}\text { Beam } \\
\left({ }^{\prime \prime}\right)\end{array}$ \\
\hline Oct. & 19 & 1992 & a & A & 1.0 & 0.4 \\
May & 7 & 1994 & b & AnB & 0.8 & 1.3 \\
Sept. & 15 & 1994 & c & BnC & 3.2 & 4.0 \\
Sept. & 28 & 1995 & d & AnB & 4.2 & 1.2 \\
Oct. & 3 & 1992 & e & D & 1.0 & 29 \\
\hline
\end{tabular}

Except for the September 1995 experiment which used 3C 48, absolute flux calibration was set using 3C 286 and the flux scale of Baars et al. (1977) as modified in the 15APR92 version of the Astronomical Image Processing System (AIPS). Phase calibrators were observed every few hours during each of the experiments.

Data reduction consisted of making tapered $180^{\prime \prime} \times 180^{\prime \prime}$ CLEANed images and using only the first clean component to phase self-calibrate the data (equivalent to using a point source model at the location of the

1 NRAO is operated by Associated Universities, Inc., under 
strongest radio source). A second untapered map, centered at the location of the peak on the first map, was made and CLEANed. The rms noise was measured in a region excluding all sources on the final map. The position and flux density of all sources whose signal-to-noise ratio exceeded 5 were recorded.

In Table 2 we present the 1861 RGB sources for which radio components were detected. We present only a sample page here; a full copy of the table is available from the CDS via anonymous ftp to cdsarc.ustrasbg.fr (130.79.128.5), via the WWW at http://cdsweb. u-strasbg.fr/Abstract.html or at ftp://ftp.astro.psu.edu /pub/edf/rgb_tab2.html and rgb_tab3.html, or by contacting the authors. The columns in Table 2 give the source name, J2000 radio position, observation code (defined in Table 1), signal-to-noise ratio, corrected $5 \mathrm{GHz}$ core VLA flux density $\left(S_{5}^{\text {core }}\right)$, total $5 \mathrm{GHz}$ Green Bank flux density taken from the GB96 catalog or from the reanalysis of the GB survey images $\left(S_{5}^{\text {tot }}\right)$, and error of the total flux density if the source appeared in GB96. We refer to individual sources using the catalog prefix "RGB J" (RASS-Green Bank catalog, J2000 epoch positions) and append "A", "B", "C", etc. to denote multiple radio sources found on a particular field.

In Table 3 we show a typical page of similar information for the 436 sources detected only at low resolution which have been excluded from further analysis. Table 4 lists the 83 fields for which no source with a signal-to-noise ratio greater than 5 was detected. Many of these are faint sources cataloged by Neumann et al. (1994) but not in GB96 which used the stricter criterion for source existence and are probably spurious. The columns list the source name, J2000 Green Bank position, observation code, total GB $5 \mathrm{GHz}$ flux density, and error in the total flux density if the source appeared in GB96. In addition, two RGB sources (RGB J0425+179, RGB J1303+488) were not observed with the VLA, but are part of the complete RGB sample.

The last column in the tables indicates the presence of a note which indicates: (1) the source may be spurious or related to a diffuse Galactic object (e.g. a supernova remnant); (2) the core radio flux density is from an observation other than this paper; or (3) the source is more than $3 \sigma$ from its GB position (Sect. 3.2).

\subsection{Flux density corrections}

Instrumental effects degrade the measured flux density for sources far from the field center. While time average smearing is insignificant for our observations, both bandwidth smearing (chromatic aberration) and primary beam degradation are significant for many sources in the RGB catalog. The corrected flux density, $S$, is given by:

$S=\frac{S^{\prime}}{B \cdot P}$ where $S^{\prime}$ is the flux density from the final map. The bandwidth smearing correction term, $B$, and the primary beam correction term, $P$, are given by (Condon et al. 1996):

$$
\begin{aligned}
& B=\left[1+\frac{2 \ln 2}{3}\left(\frac{\Delta \nu \rho}{\nu \Theta_{0}}\right)^{2}\right]^{-\frac{1}{2}} \text { and } \\
& P=\left(a_{0}+a_{1} x+a_{2} x^{2}+a_{3} x^{3}+a_{4} x^{4}\right)^{-1} .
\end{aligned}
$$

Here $\Delta \nu$ is the bandwidth $(50 \mathrm{MHz}), \nu$ is the observing frequency $(4.885 \mathrm{GHz}), \rho$ is the angular distance from the field center, $\Theta_{0}$ is the restoring beam size (Table 1), $x=(\rho \nu)^{2}(\rho$ in arcminutes $), a_{0}=1.003, a_{1}=1.08610^{-3}$, $a_{2}=3.3010^{-6}, a_{3}=-3.60910^{-9}$ and $a_{4}=3.30510^{-12}$.

\subsection{Source parameter reliability}

While the formal uncertainties for our reported flux densities and positions can be defined as a quadratic sum of the squares of several error terms (e.g. Condon et al. 1982; Kollgaard et al. 1994), we found these formal uncertainties underestimated the true uncertainties in the reported source parameters. The biggest sources of error in the RGB catalog are instead due to instrumental and technical effects intrinsic to our snapshot mode. In order to assess the reliability in our measured flux densities and positions, we observed 20 RGB sources at more than one epoch after the main survey was completed and used the same data reduction procedure to obtain core flux densities and positions.

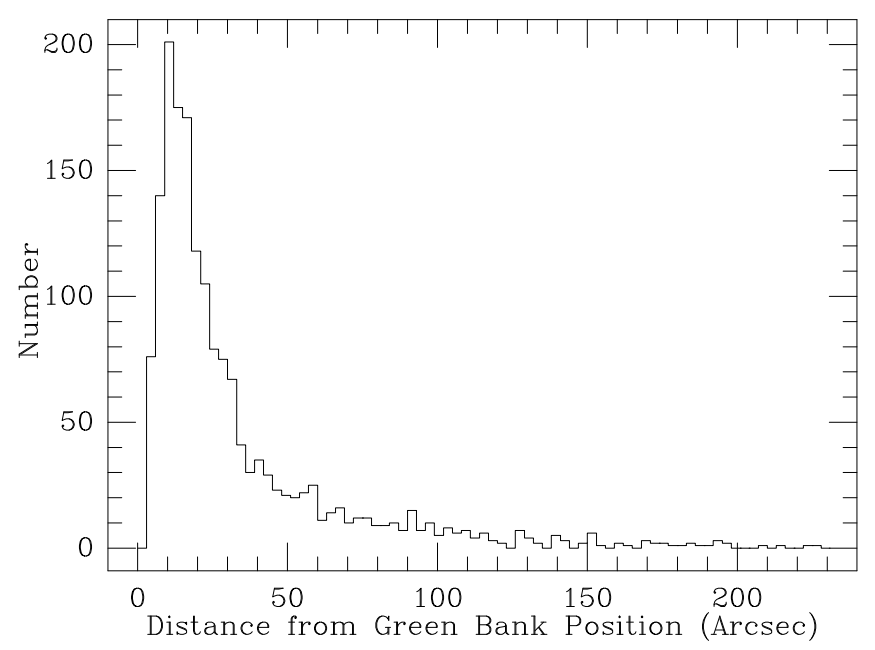

Fig. 1. Distribution of VLA - Green Bank radio positions

These repeated observations show the reported positions for sources in Table 2 are accurate to $\leq 0.5^{\prime \prime}$ while those in Table 3 are accurate to $\leq 8^{\prime \prime}$. The core flux densities of the sources observed at multiple epochs varied significantly, however, with the source intensity varying 


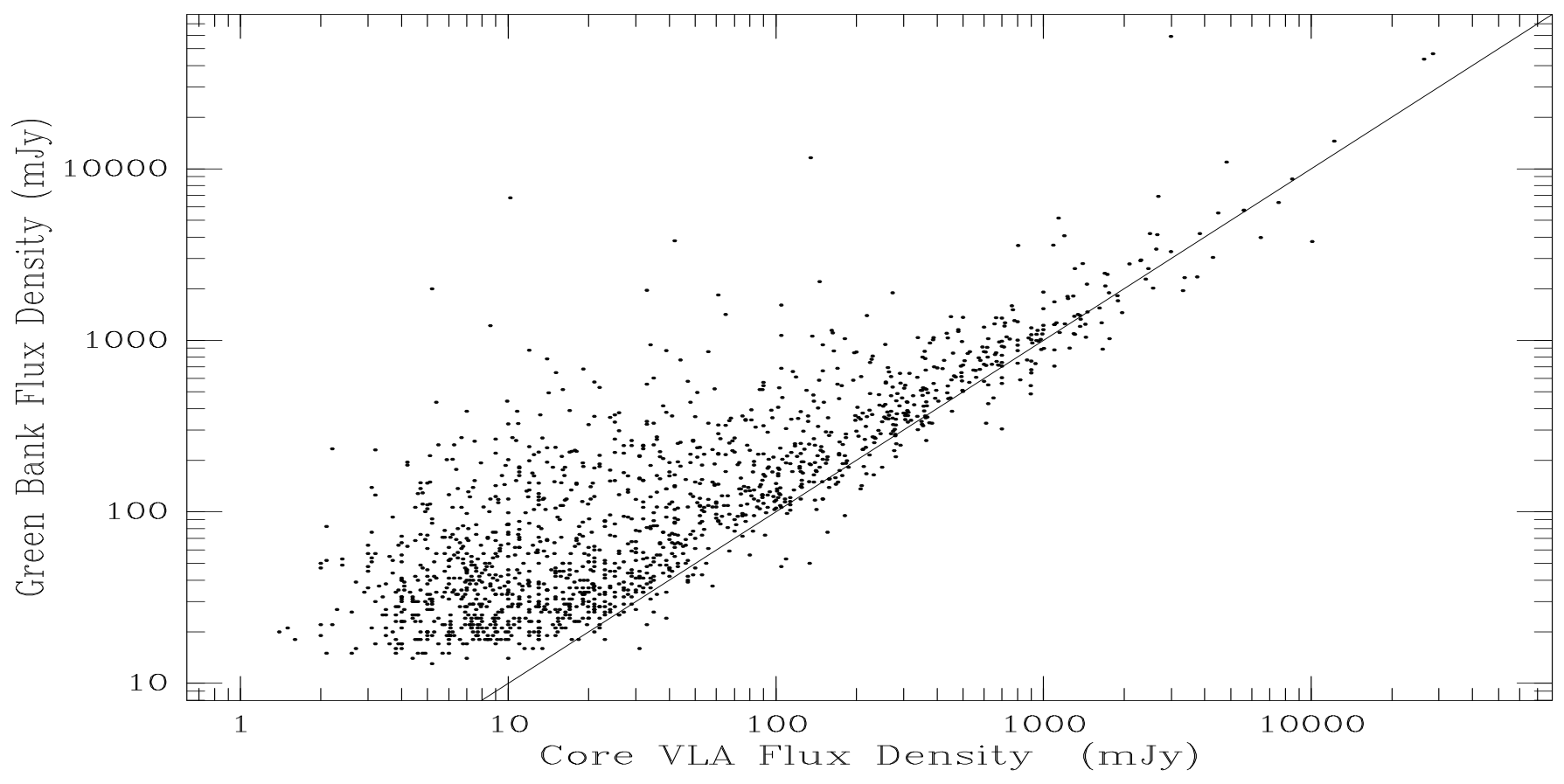

Fig. 2. Log - Log diagram of the core VLA radio flux density (mJy) vs. Green Bank flux density (mJy) at $5 \mathrm{GHz}$

by as much $80 \%$ between epochs separated by as little as 10 days. While some of this variability may be intrinsic to the sources, we believe much of it is due to instrumental causes such as different VLA resolution, the lack of phase calibrators near individual sources, very short observation times, and consequently the small number of visibilities used to image large fields. Our tests show the reported flux densities of sources fainter than $\sim 20$ mJy are generally accurate to $\sim 50 \%$ and the brighter sources accurate to $\sim 20 \%$.

\section{Comparison of the Green Bank and VLA source properties}

Figure 1 shows that $82 \%$ of the sources are within $\sim 50$ " of the expected GB96 position and the distribution peaks at an offset of $10^{\prime \prime}-15^{\prime \prime}$, consistent with the positional errors given in Neumann et al. (1994) and Gregory \& Condon (1991). Because the positional uncertainty of the Green Bank positions (which greatly dominate the uncertainties in the VLA positions) are flux dependent, we split the data into two subsets: a bright and faint sample with a division at $75 \mathrm{mJy}$. We find that the positional accuracies given in Neumann et al. (1994) are then reliable for these two samples. For the analyses that follow, we therefore exclude all bright sources offset from the GB positions by more than $45^{\prime \prime}$ and all faint sources offset by more than $120^{\prime \prime}$. These criteria exclude 74 objects from the bright sample and 47 objects from the faint. The number of spurious coincidences which remain in the tables is quite small with only $\sim 31$ of the 757 remaining faint sources and $\sim 10$ of the 813 remaining bright sources expected to be spurious. Flags are given in Table 2 to indicate the sources we excluded.

Figure 2 shows the flux-flux diagram comparing the core VLA flux density with the Green Bank measurement. For those $\sim 10 \%$ of the fields for which more than one source was detected, we have chosen as the "core" component that source which is closest to the RASS position. We also considered using the brightest source on the field or the source closest to the GB position. In all the following analyses, the differences between the results obtained using these three different criterion are well within the uncertainties in the data. Only when we completely excluded the fields with more than one detected source did any of the results change significantly, increasing the median core-to-lobe parameter (Sect. 5 ) by $\sim 20 \%$. While source variability and uncertainties in both flux density measurements produces a few points in Fig. 2 where $S_{5}^{\mathrm{VLA}}>S_{5}^{\mathrm{GB}}$, it is clear that nearly all of the sources in the GB catalog contain emission which is significantly resolved at the arcsecond-scale.

\section{Radio beaming}

The radio core-to-lobe ratio, $R=S_{5}^{\text {core }} / S_{5}^{\text {lobe }}$, is often used in studies of radio-loud AGN as a relative measure of orientation (e.g. Orr \& Browne 1982). In the absence of high quality interferometric maps showing full details of the radio structure, this ratio can be approximated as $R^{\prime}=S_{5}^{\mathrm{VLA}} /\left(S_{5}^{\mathrm{GB}}-S_{5}^{\mathrm{VLA}}\right)$. The distribution of $R^{\prime}($ Fig. $3 \mathrm{a})$ will differ in detail from that of the true $R$, both because 

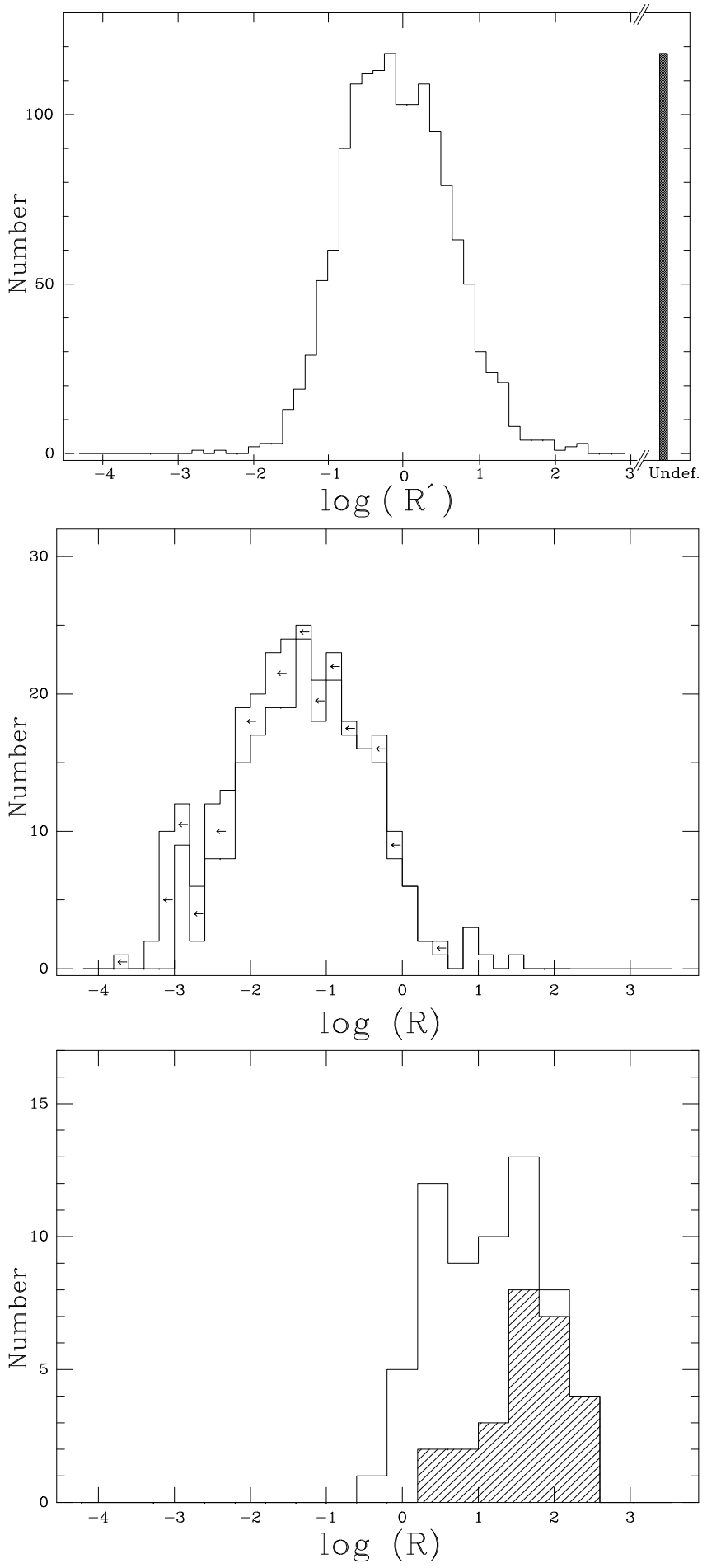

Fig. 3. a) Distribution of $R^{\prime}$, defined as the (VLA core flux density)/(Green Bank flux density - VLA core flux density). This parameter is a rough measure of the radio core-to-lobe ratio. The last bin contains the 118 sources for which the Green Bank flux density is less than the VLA core flux density. b) Distribution of the core-to-lobe ratio for radio galaxies. Bins containing undetected cores are denoted with a left-facing arrow. c) Distribution of the core-to-lobe ratio for BL Lacertae objects. The hatched histogram distinguishes the radio-selected from the X-ray-selected objects the VLA and GB observations were not simultaneous and because the low resolution GB measurement may contain some emission from unrelated sources. Nevertheless, we believe these effects should not introduce any significant biases into the distribution of $R^{\prime}$.

For comparison we show in Fig. 3b the distribution of core-to-lobe ratios for a large sample of FRI and FRII radio galaxies compiled by Zirbel \& Baum (1995). (See Zirbel \& Baum 1995 for a discussion of the assumptions used to derive $R$ and the upper limits on $R$ for those sources without a measured radio core.) To illustrate the properties of an extremely core-dominated population, we show in Fig. 3c the core-to-lobe ratio for BL Lacertae objects, with the radio-selected objects (RBLs) represented by the hatched histogram. The objects shown are the X-ray-selected BL Lacs (XBLs) from the HEAO1 Large Area Sky Survey (Kollgaard et al. 1996) and the Einstein Extended Medium Sensitivity Survey (Morris et al. 1991), and the radio-selected BL Lacs in the 1 Jansky sample (Stickel et al. 1991). The radio flux densities used to derive the core-to-lobe ratios were taken from Kollgaard et al.

Figure 3 shows the RGB sample is more coredominated ( $40 \%$ of the sources have $\log R^{\prime}>0$ ) than the radio galaxy sample of Zirbel \& Baum (1995; 3\% with $\log R>0$ ), but is less core-dominated than the BL Lacertae objects ( $82 \%$ with $\log R>0)$. We used the Astronomy SURVival (ASURV) data analysis software (Rev. 1.2; LaValley et al. 1992) to compute the KaplanMeier estimator of the $R$ distributions. This properly takes into account the upper limits in the radio galaxy sample (Feigelson \& Nelson 1985). The median $R$ of each distribution is given in Table 5 . We find that both classes of BL Lac objects are significantly more core-dominated than the RGB sample. The median of the radio galaxy sample, however, is 27 times less core-dominated than the RGB sources.

The differences discussed above are clearly due to the type of object which dominates each of the samples. Although $>70 \%$ of the RGB catalog is optically unidentified, most of the identified sources are quasars (B95). A comparison of the optically identified and unidentified sources shows that while the identified sources generally exhibit higher radio and X-ray fluxes, other properties (e.g. their optical colors) are not statistically different (B96). This suggests the unidentified sources are also primarily quasars. The differences in the distribution of $R$ therefore indicate the RGB catalog consists primarily of quasars whose radio emission is moderately beamed.

Within the framework of the unified scheme scenario which hypothesizes flat and steep spectrum quasars are radio galaxies seen close to the line-of-sight (e.g. Barthel 1989), we use a simple beaming model and the core-tolobe ratio distributions to constrain the jet speed and orientation characteristic of objects in the RGB sample. The 
dependence of $R$ on jet speed and orientation are given by (e.g. Urry \& Padovani 1995):

$R \equiv \frac{S_{\mathrm{r}}^{\text {core }}}{S_{\mathrm{r}}^{\text {lobe }}}=f \delta^{p}$

where $f$ is the intrinsic core-to-lobe ratio, $p$ is the beaming index, and $\delta$ is the Doppler factor:

$\delta=[\Gamma(1-\beta \cos \theta)]^{-1}$.

Here $\beta=v / c$, where $v$ is the bulk velocity, $\Gamma=\left(1-\beta^{2}\right)^{-\frac{1}{2}}$, and $\theta$ is the angle to the line-of-sight. We assume $p=2.7$, applicable to a jet consisting of a single sphere with a spectral index $\alpha=0.3\left(S_{\nu} \propto \nu^{\alpha}\right.$; e.g. Pearson \& Zensus 1987). We make the further assumption that the Zirbel \& Baum (1995) sample of FRI and FRII radio galaxies is characteristic of the parent population of RGB sources, although we examine this hypothesis more carefully at the end of this section.

Table 5. Median core-to-lobe ratios

\begin{tabular}{ll}
\hline \multicolumn{1}{c}{ Sample } & \multicolumn{1}{c}{$R$} \\
\hline Radio galaxies & 0.019 \\
RGB sample & 0.52 \\
XBLs & 1.8 \\
RBLs & 20. \\
\hline
\end{tabular}

Kollgaard et al. (1996), analyzing the same population of radio galaxies and $\mathrm{BL}$ Lacertae objects, found that the relative core enhancement of these populations implied that $\Gamma>4.5$ and probably exceeded $\Gamma=6$. We therefore assume initially $\Gamma=6$ for all three populations and adopt $\bar{\theta}=60^{\circ}$ for the radio galaxies. (See Kollgaard et al.) For a sample like the RGB catalog which consists largely of radio-loud quasars (B95; B96; Laurent-Muehleisen et al., in preparation), the assumption $\Gamma=6$ is a reasonable lower limit to the jet speed (Urry \& Padovani 1995). Using these assumptions and the median $R$ values in Table 5 , this implies that the average angle to the line-of-sight for the RGB sample $\left(\bar{\theta}_{\mathrm{RGB}}\right)$ is approximately $30^{\circ}$, significantly larger than that obtained for the BL Lac objects where $\bar{\theta}_{\mathrm{XBL}} \approx 20^{\circ}$ and $\bar{\theta}_{\mathrm{RBL}} \approx 10^{\circ}$ (Kollbaard et al. 1996).

The assumption that $\Gamma$ is a single value is most likely incorrect in detail since a range of jet speeds probably characterizes any given population of objects. Assuming $\bar{\theta}_{\text {gal }}=60^{\circ}$ and $\Gamma_{\mathrm{RGB}}=\Gamma_{\text {gal }}$, but allowing both Lorentz factors to vary over the range $2 \geq \Gamma \geq 20$, constrains the average angle to the line-of-sight for the RGB sample to lie within a fairly small range, $20^{\circ}<\bar{\theta}_{\mathrm{RGB}}<32^{\circ}$ (Fig. 4). If we further constrain $\Gamma \geq 5$, which is a reasonable minimum based on studies of the observed luminosity function of flat

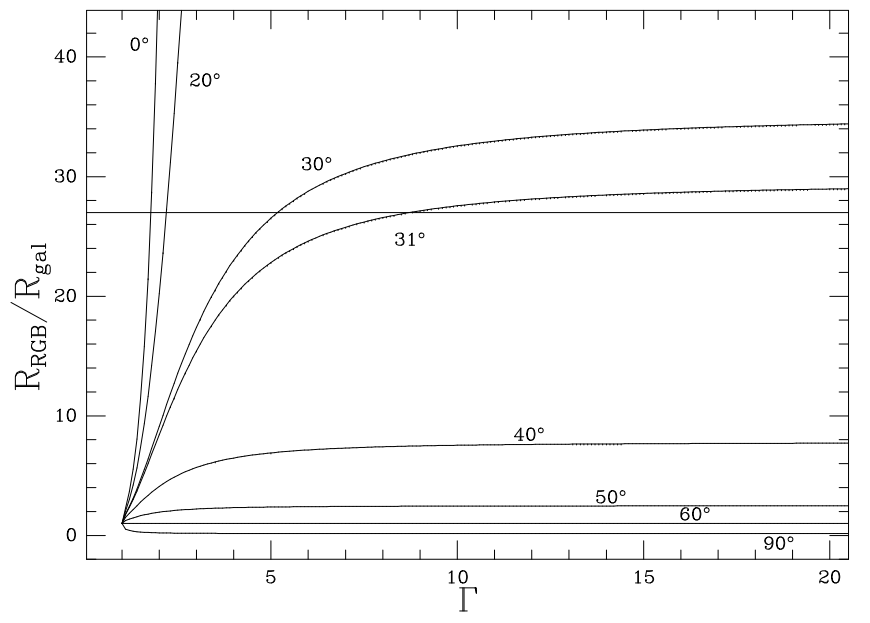

Fig. 4. The predicted line-of-sight orientation for the RGB sample, $\bar{\theta}_{\mathrm{RGB}}$, derived from the relative median core-to-lobe ratios of the RGB and radio galaxy samples. $\bar{\theta}_{\text {gal }}=60^{\circ}$ is assumed and the horizontal line is the observed ratio of $\frac{R_{\mathrm{RGB}}}{R_{\text {gal }}}$

and steep spectrum quasars (Urry \& Padovani 1995), then $\bar{\theta}_{\mathrm{RGB}}$ is narrowly confined to be about $31^{\circ}$.

Finally, we consider the possibility that the population of FRI and FRII radio galaxies used here is not characteristic of the parent population of objects in the RGB catalog. Assuming some form of a unified scheme is not unreasonable, but it is possible that the RGB sample exhibits an average jet speed substantially different than that characteristic of the Zirbel \& Baum (1995) radio galaxy sample. This could be the case if the RGB catalog is biased toward objects with a larger $\Gamma$. Using the results of Kollgaard et al. (1996), we fix $\Gamma_{\text {gal }}=6$ and $\bar{\theta}_{\text {gal }}=60^{\circ}$. As before, we constrain $\Gamma_{\mathrm{RGB}}$ to be larger than 5 . The average angle to the line-of-sight for the RGB sample is then $20^{\circ} \geq \bar{\theta}_{\mathrm{RGB}} \geq 35^{\circ}$. We also consider the case where the intrinsic core-to-lobe ratio ( $f$ in Eq. (4)) of the Zirbel \& Baum (1995) radio galaxies is different than that of the "true" parent population. Since the RGB catalog is likely dominated by radio- and X-ray-loud quasars, if the FRII-quasar unified scheme is correct (Barthel 1989), the true parent population of RGB objects will have extended radio powers approximately two to three orders of magnitude higher than those objects in Zirbel \& Baum (1995). Because the core-to-lobe ratio decreases with increasing extended radio power (Kollgaard et al. 1996), our ratio of the core-to-lobe parameters would be too low by a factor of $\sim 4$. However, the effect on the average angle to the line-of-sight is fairly modest, decreasing it to $\bar{\theta}_{\mathrm{RGB}} \sim 25^{\circ}$. These considerations indicate that $\bar{\theta}_{\mathrm{RGB}}$ is relatively insensitive to assumptions about the detailed characteristics of the parent population. 

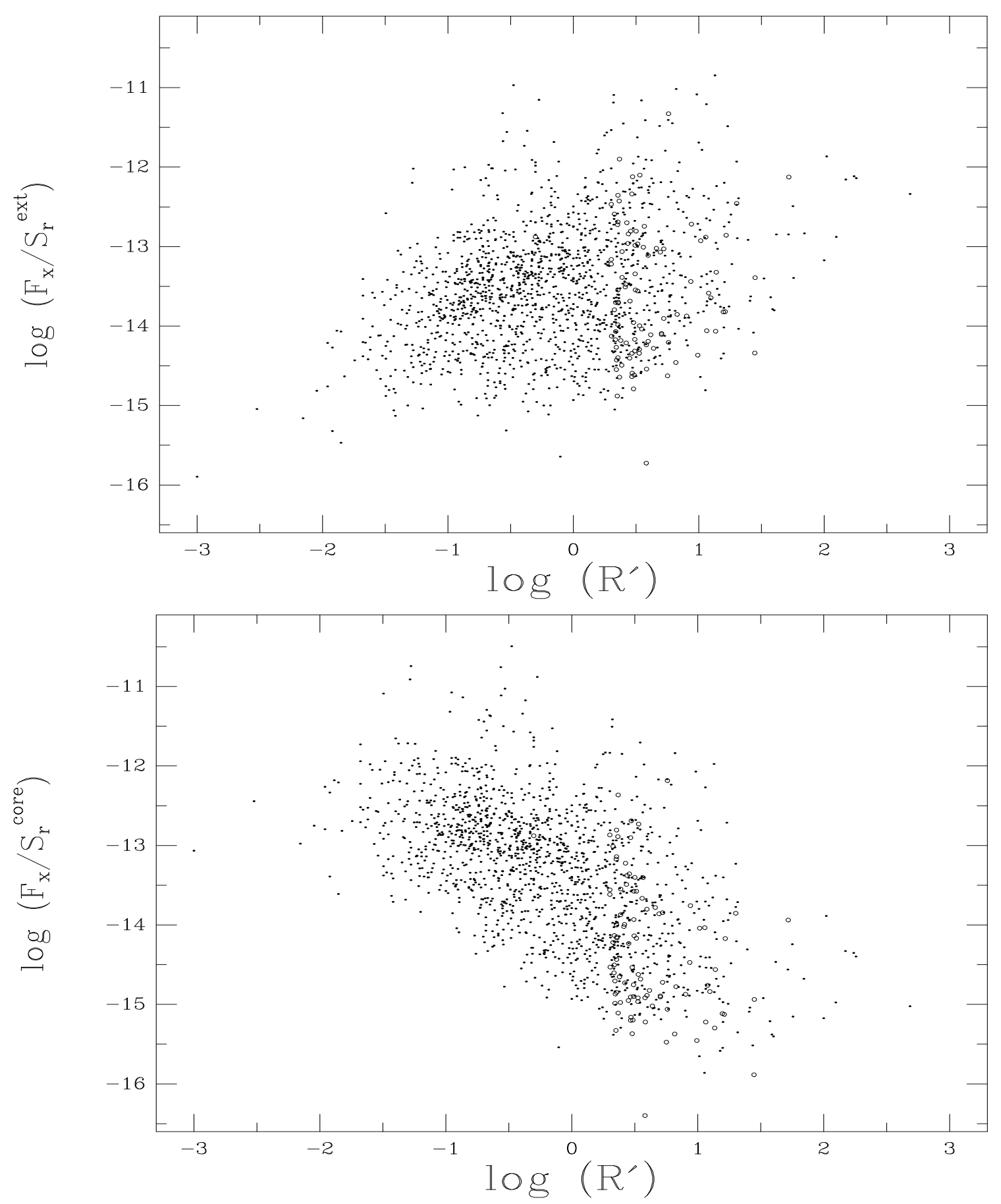

Fig. 5. a) The ratio of $X$-ray to extended radio flux density as a function of the radio core-to-lobe ratio, $R^{\prime}$, for the $R G B$ sources. For those 118 sources for which the VLA core flux density was greater than the Green Bank total flux density, we derive upper limits as described in the text. These points are denoted by open circles in the diagram. b) Similar to a) except the ordinate shows the ratio of X-ray to core radio flux density 


\section{X-ray beaming?}

While the radio emission from radio-loud AGN consists of both beamed (core) and unbeamed (extended) components, the origin of the X-ray emission is not as clear. Only recently has the deconvolution of thermal (host galaxy) $\mathrm{X}$-ray emission from nonthermal unresolved emission been possible (e.g. Worrall \& Birkinshaw 1994). Although these studies are preliminary and the sample sizes small, it is reasonable to assume the X-ray fluxes for the RGB sample likely consist of a heterogeneous mix of these two components. General trends should nevertheless persist in the data revealing how much, if any, of the X-ray emission is beamed.

Because redshifts are not available for most of the objects in the RGB sample, flux ratios must be used to compensate for distance effects. Specifically, we compare the ratio of the X-ray flux $\left(F_{\mathrm{x}}\right.$, in erg s $\left.\mathrm{s}^{-1} \mathrm{~cm}^{-2}\right)$ to the beamed radio core $\left(S_{\mathrm{r}}^{\text {core }}\right.$ in mJy) and unbeamed extended $\left(S_{\mathrm{r}}^{\text {ext }}\right)$ components, with the radio core-to-lobe ratio $R^{\prime}$ (Fig. 5). For those sources where our VLA core measurement exceeded the GB total flux density measurement, we derive limits (in both the ordinate and abscissa) by assuming the maximum uncertainty in the radio flux densities, namely $20 \%$ error for sources $>20$ mJy and $50 \%$ error otherwise. (See Sect. 3.2.) It is likely that the uncertainty in the measurements and not source variability are to blame since most of the sources which suffer from this effect are faint and therefore have the greatest uncertainty in their measured flux densities. Nevertheless, because there are so many sources where $S_{\mathrm{r}}^{\text {ext }} \geq S_{\mathrm{r}}^{\text {core }}$, the statistical significance of the following analyses remains unchanged whether the upper limits are halved or doubled, thus insuring our results are insensitive to our particular method for computing the limits.

Figure 5a shows the ratio of the total X-ray flux to extended radio flux density $\left(F_{\mathrm{x}} / S_{\mathrm{r}}^{\text {ext }}\right)$ versus $R^{\prime}$ while in Fig. 5b we show the ratio of the total X-ray flux to core radio flux density $\left(F_{\mathrm{x}} / S_{\mathrm{r}}^{\text {core }}\right)$ versus $R^{\prime}$. Two trends are seen in Fig. 5: $F_{\mathrm{x}} / S_{\mathrm{r}}^{\text {ext }}$ increases with increasing $R^{\prime}$, and $F_{\mathrm{x}} / S_{\mathrm{r}}^{\text {core }}$ decreases with increasing $R^{\prime}$. Both these trends are statistically significant at the $>99.99 \%$ level. Two possible biases could affect the correlations. First, if the 83 sources for which no arcsecond-scale source was detected (Table ??) are real and not spurious detections in the $3 \sigma$ Green Bank catalog, they must be lobe-dominated and would appear on the left-hand side of Fig. 5a. If these sources were also systematically X-ray brighter so that they had high $F_{\mathrm{x}} / S_{\mathrm{r}}^{\text {ext }}$ ratios then they could populate the upper left portion of Fig. 5a; however, the ROSAT fluxes for these sources span the same range as the detected sources, indicating this potential bias is not present. Second, the different flux limits of the original GB cat$\operatorname{alog}\left(S_{\mathrm{r}}^{\text {tot }} \geq 15 \mathrm{mJy}\right)$ and the deeper VLA core measurements $(\sim 1 \mathrm{mJy})$ imply core-dominated sources with $1 \leq S_{\mathrm{r}}^{\text {tot }} \leq 15 \mathrm{mJy}$ are missing from the RGB catalog.
These "missing" sources could destroy the correlation in Fig. 5b only if their core-to-lobe ratios exceeded $\sim 10.0$ and $F_{\mathrm{x}}$ exceeded $\sim 10^{-13} \mathrm{erg} \mathrm{s}^{-1} \mathrm{~cm}^{-2}$. This is not the case, however, since our VLA flux limit is only one order of magnitude deeper than the GB flux limit thereby constraining the core-to-lobe ratios of the missing sources to be $-1.0<\log R^{\prime}<1.0$. We therefore conclude that the two trends in Fig. 5 are real.

To understand these relationships, we characterize the $\mathrm{X}$-ray emission by an X-ray "core-to-extended" ratio, $R_{\mathrm{X}}$, defined by:

$R_{\mathrm{X}} \equiv \frac{F_{\mathrm{x}}^{\text {core }}}{F_{\mathrm{x}}^{\text {ext }}}$

If the X-ray core beaming is simply related to the radio core beaming, we can write $R_{\mathrm{X}}=k R^{\prime}$ where $k$ is a constant. The quantities plotted in Fig. 5 are then:

$\frac{F_{\mathrm{x}}}{S_{\mathrm{r}}^{\text {core }}}=\frac{F_{\mathrm{x}}^{\mathrm{ext}}}{S_{\mathrm{r}}^{\text {ext }}} \frac{1+k R^{\prime}}{R^{\prime}}$

and

$\frac{F_{\mathrm{x}}}{S_{\mathrm{r}}^{\mathrm{ext}}}=\frac{F_{\mathrm{x}}^{\mathrm{ext}}}{S_{\mathrm{r}}^{\text {ext }}}\left(1+k R^{\prime}\right)$

First we consider the case where the $\mathrm{X}$-ray emission is isotropic so that $F_{\mathrm{x}}=F_{\mathrm{x}}^{\text {ext }}$ and $k=0$. Then as the angle to the line-of-sight decreases, both $R^{\prime}$ and $S_{\mathrm{r}}^{\text {core }}$ increase but $F_{\mathrm{x}}$ remains constant. The ratio of $F_{\mathrm{x}} / S_{\mathrm{r}}^{\text {core }}$ would therefore be anticorrelated with $R^{\prime}$ as seen in Fig. 5b. However, the ratio of $F_{\mathrm{x}} / S_{\mathrm{r}}^{\text {ext }}$ would be uncorrelated with $R^{\prime}$ since neither parameter would vary with orientation. The positive correlation in Fig. 5a, therefore rules out the possibility that X-ray emission for sources in the RGB catalog is entirely isotropic.

If we now consider the other extreme where the X-ray emission consists of a much higher fraction of beamed radiation than the radio emission $(k>>1) . F_{\mathrm{x}} / S_{\mathrm{r}}^{\text {ext }}$ should then be correlated with $R^{\prime}$, as observed, but the ratio of $F_{\mathrm{x}} / S_{\mathrm{r}}^{\text {core }}$ would become uncorrelated with $R^{\prime}$ at even modest values of $R^{\prime}$, which is clearly not seen (Fig. $5 \mathrm{~b}$ ). Therefore, if the X-ray emission is beamed, it is not characterized by a high $k$-value. As an alternative to the models presented in Eqs. (6-8), we consider the case where $\Gamma_{\mathrm{x}} \neq \Gamma_{\mathrm{r}}$. Such a scenario has been proposed in terms of an accelerating jet model for BL Lac objects (e.g. Ghisellini \& Maraschi 1989) where $\Gamma_{\mathrm{x}} \leq \Gamma_{\mathrm{r}}$. We find that in order to produce the relations seen in Fig. 5 which are valid over three orders of magnitude in the $\mathrm{X}$-ray to radio flux ratios and five orders of magnitude in $R^{\prime}, \Gamma_{\mathrm{x}} \geq 4$ and $\Gamma_{\mathrm{r}} \geq 6$, which is consistent with bulk velocities inferred through other means (e.g. Urry \& Padovani 1995).

Figures $5 \mathrm{a}$ and $5 \mathrm{~b}$ therefore indicate the X-ray emission of the RGB sample is neither entirely isotropic $\left(R_{\mathrm{X}}=0\right)$ nor characterized by a high $k$-value. However, the scatter in the diagrams is large enough to prevent an 
accurate measurement of the fraction of beamed X-rays or even to distinguish beamed X-ray emission from unbeamed but anisotropic emission. The latter could arise, for example, from a population of objects with an obscuring torus with varying column density which blocks more soft X-rays as the torus becomes more edge-on to the lineof-sight.

The large scatter in the diagrams is primarily due to the heterogeneity of the RGB sample, which includes radio galaxies, quasars and BL Lacs. Examination of a single class of AGN, such as RGB BL Lacs, can yield more quantitative results (Laurent-Muehleisen et al., in preparation).

\section{Conclusions}

We present subarcsecond radio positions and core radio flux densities for all 2,127 sources appearing in both the Green Bank $5 \mathrm{GHz}$ and in the ROSAT All-Sky Survey catalogs. The accuracy of the positions is sufficient to give unique optical identifications for the X-ray- and radioemitting sources (B96).

This RGB sample is comprised principally of radioloud AGN. It is complete and unbiased with well-defined selection criteria: X-ray flux above the RASS sensitivity limit (which depends only on ecliptic latitude), arcminutescale radio flux density above $\simeq 15 \mathrm{mJy}$ at $5 \mathrm{GHz}$, and declination between $0^{\circ}$ and $75^{\circ}$.

The radio emission of the $\mathrm{RGB}$ sample is found to be more core-dominated than ordinary radio galaxies but less than strongly beamed BL Lac objects, which suggests it consists primarily of moderately beamed AGN. Using simple beaming models, the typical RGB object is shown to be dominated by a jet oriented at an intermediate angle to the line-of-sight $\left(\bar{\theta}_{\mathrm{RGB}} \sim 25^{\circ}-35^{\circ}\right)$. The X-ray beaming properties are not tightly constrained, but exclude the extremes of purely isotropic emission and of X-ray emission which consists of a significantly higher fraction of beamed flux than is characteristic of the radio emission.

Note in press: After submission of this paper, we discovered a possible systematic position error in a very small subset of sources. In particular, the sources RGB J0131+005, RGB J0139+178, RGB J0143+129, RGB J0157+235A, RGB J0232+202, RGB J0233+024A, RGB J0243+171, RGB J0256+035, RGB J0303+059, RGB J0308+104, RGB J0312+243A, RGB J0312+242C, and RGB J0314+063 were all observed in our "c" epoch VLA runs and show large systematic offsets with respect to sources found in the NVSS survey. We re-examined these sources and found the position reported in the tables is accurate given our data, but that the noise on these fields before any CLEAN-ing is abnormally high. We thank Dr. Alastair Edge for pointing out this discrepancy.
Acknowledgements. We wish to thank C. Palma for help with the data reduction at Penn State. This work was partially supported by NASA under Grant NAGW-2120. We have made use of the NASA/IPAC Extragalactic Database, operated by the Jet Propulsion Laboratory, California Institute of Technology, under contract with NASA.

\section{References}

Baars J.W.M., Gnezel R., Pauliny-Toth I.I.K., Witzel A., 1977, A\&A 61,99

Bade N., Fink H.H., Engels D., Voges W., Hagen H.J., Wisotzki L., Reimers D., 1995, A\&AS 110, 469

Barthel P.D., 1989, ApJ 336, 606

Baum S.A., Heckman T., 1989, ApJ 336, 681

Becker R.H., White R.L., Edwards A.L., 1991, ApJS 75, 1

Brinkmann W., Siebert J., Boller Th., 1994, A\&A 281, 355

Brinkmann W., Siebert J., Reich W., et al., 1995, A\&AS 109, 147 (B95)

Brinkmann W., Siebert J., Feigelson E.D., et al., 1997, A\&A (submitted; B96)

Browne I.W.A., Murphy V., 1987, MNRAS 226, 601

Burns J.O., Basart J.P., De Young D.S., Ghiglia D.C., 1984, ApJ 283, 515

Condon J.J., Condon M.A., Hazard C., 1982, AJ 87, 739

Condon J.J., Cotton W.D., Greisen E.W., Yin Q.F., Perley R.A., Broderick J.J., 1996, AJ (in preparation)

Elvis M., Plummer D., Schachter J., Fabbiano G., 1992, ApJS 80,257

Feigelson E.D., Isobe T., Kembhavi A., 1984, AJ 89, 1464

Feigelson E.D., Nelson P.I., 1985, ApJ 293, 192

Ghisellini G., Maraschi L., 1989, ApJ 340, 181

Ghisellini G., Padovani P., Celotti A., Maraschi L., 1993, ApJ 407, 65

Gioia I., Maccacaro T., Schild R., Wolter A., Stocke J., Morris S., Henry J.P., 1990, ApJS 72, 567

Gower A.C., Hutchings A.C., 1984, AJ 89, 1658

Gregory P.C., Condon J.J., 1991, ApJS 75, 1011

Gregory P.C., Scott W.K., Douglas K., Condon J.J., 1996, ApJS 103, 427 (GB96)

Hintzen P., Ulvestad J., Owen F., 1983, AJ 88, 709

Hutchings J.B., Price R., Gower A.C., 1988, ApJ 329122

Kellerman K.I., Sramek R.A., Schmidt M., Shaffer D.B., Green R.F., 1989, AJ 98, 1195

Kollgaard R.I., Wardle J.F.C., Roberts D.H., Gabuzda D.C., 1992, AJ 104, 1687

Kollgaard R.I., Brinkmann W., Chester M.M., et al., 1994, ApJS 93, 145

Kollgaard R.I., Palma C., Laurent-Muehleisen S.A., Feigelson E.D., 1996, ApJ 465, 115

Laurent-Muehleisen S.A., Kollgaard R.I., Ciardullo R.B., et al., 1996 (in preparation)

Lawson A.J., Turner M.J.L., Williams O.R., Stewart G.C., Saxton R.D., 1992, MNRAS 259, 743

LaValley M., Isobe T., Feigelson E.D., 1992, BAAS 24, 839

Linfield R., Perley R., 1984, ApJ 279, 60

Miller P., Rawlings S., Sanders R., 1993, MNRAS 263, 425

Morris S.L., Stocke J.T., Gioia I.M., Schild R.E., Wolter A., Maccacaro T., Della Ceca R., 1991, ApJ 380, 49

Murphy D.W., Browne I.W.A., Perley R.A., 1993, MNRAS 264,298 
Neumann M., Reich W., Fürst E., Brinkmann W., Reich P., Siebert J., Wielebinski R., Trümper J., 1994, A\&AS 106, 303

O’Dea C.P., Owen F.N., 1985, AJ 90, 927

Orr M.J., Browne I.W.A., 1982, MNRAS 200, 1067

Pearson T.J., Zensus J.A., 1987, in Superluminal Radio Sources, Zensus J.A. \& Pearson T.J. (eds.). Cambridge University Press, Cambridge, p. 1

Perley R.A., 1982, AJ 87, 859

Punsly B., 1995, AJ 109, 1555

Stickel M., Padovani P., Urry D.M., Fried J.W., Kühr H., 1991, ApJ 374, 431

Stocke J.T., Morris S.L., Gioia I.M., Maccacaro T., Schild R., Wolter A., Fleming T.A., Henry J.P., 1991, ApJS 76, 813

Ulvestad J.S., Wilson A.S., 1984, ApJ 278, 544

Urry C.M., Padovani P., 1995, PASP 107, 803

Voges W., Gruber R., Paul J., et al., 1992, The ROSAT Standard Analysis Software System, ESA ISY-3, p. 223

Voges W., 1993, Adv. Space Res. 13, 391

Wood K.S., et al., 1984, ApJS 56, 507

Worrall D.M., Birkinshaw M., 1994, ApJ 427, 134

Wrobel J.M., Heeschen D.S., 1984, ApJ 287, 41

Zirbel E.L., Baum S.A., 1995, ApJ 448, 521

\section{Notes to Table 2}

$1545+646 \mathrm{~A}$ : More than $3 \sigma$ from GB position

$1545+646 \mathrm{~B}$ : More than $3 \sigma$ from GB position

1547+208: Core radio flux from Miller et al. (1993)

1549+026: Core radio flux from Murphy et al. (1993)

1550+113: Core radio flux from Hintzen et al. (1983)

1604+012: Core radio flux from Baum \& Heckman (1989)
1608+104: Core radio flux from Murphy et al. (1993)

1609+179: Core radio flux from Hutchings et al. (1988)

$1617+350$ : More than $3 \sigma$ from GB position

1620+176: Core radio flux from Hintzen et al. (1983)

$1625+268$ : Core radio flux from Feigelson et al. (1984)

\section{Notes to Table 3}

$0242+083$ : More than $3 \sigma$ from GB position

\section{Notes to Table 4}

0140+087: Possibly Spurious Source; See Condon et al. (1994)

0205+648: Extended Source

0247+187: Extended Source

$0317+415$ : Confused Field - Possibly Spurious Source

0528+344: Extended Source

0533+210: Confused Field - Possibly Spurious Source

$0535+222$ : Confused Field - Possibly Spurious Source

0641+080: Extended Source

1232+123: Confused Field - Possibly Spurious Source

1728+086: Confused Field - Possibly Spurious Source

1852+006: Extended Source (known SNR)

1859+071: Confused Field or Extended Source

2015+386: Confused Field - Possibly Spurious Source

2212+589: Extended Source

$2302+587$ : Extended Source

2323+584: Confused Field - Possibly Spurious Source 
Table 2. Radio source properties (sample page)

\begin{tabular}{|c|c|c|c|c|c|c|}
\hline 䑃 & $* *$ & & * & & * & $\underset{4}{4}$ \\
\hline $\begin{array}{ll}0 \\
0 \\
20 \\
4 \\
4\end{array}$ & 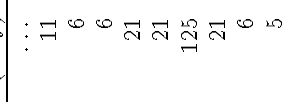 & 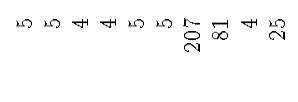 & 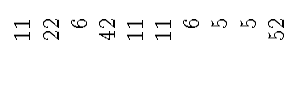 & 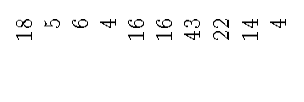 & $\vec{\sim} \stackrel{\sigma}{\rightarrow}: \backsim+\underset{\sim}{\infty}+\underset{\sim}{\infty} \cdots$ & $\stackrel{\overrightarrow{2}}{\equiv}$ \\
\hline & 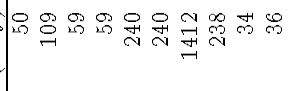 & 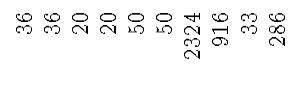 & 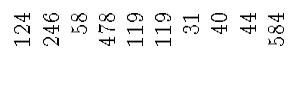 & 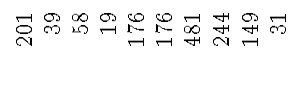 & 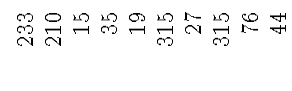 & $\overrightarrow{\tilde{\sigma}_{2}}$ \\
\hline & 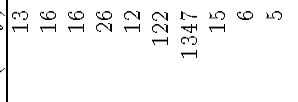 & 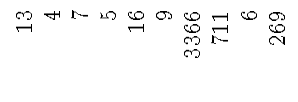 & অே & 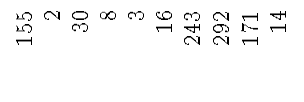 & 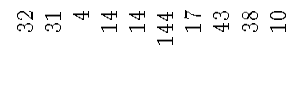 & 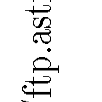 \\
\hline$\frac{z}{m}$ & 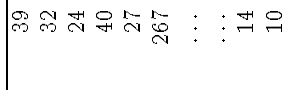 & 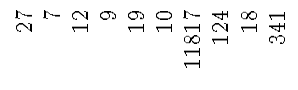 & 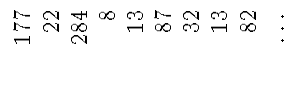 & 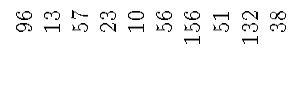 & 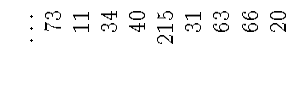 & $\ddot{\theta}_{\rightleftarrows}$ \\
\hline है & a. & $\operatorname{sen} \theta \pi \theta 0 \theta 0$ & 5. & 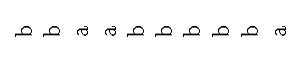 & 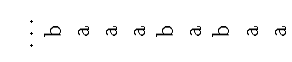 & \& \\
\hline & 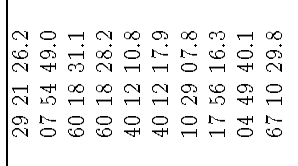 & 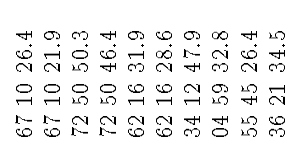 & 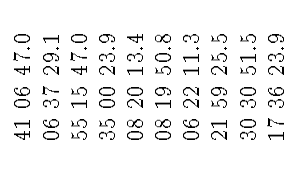 & 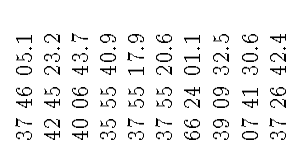 & 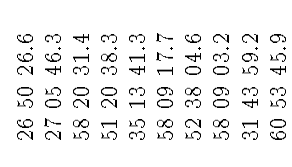 & 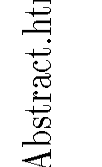 \\
\hline & 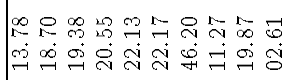 & 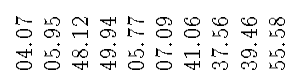 & 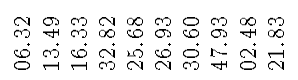 & 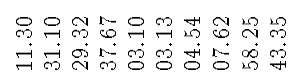 & 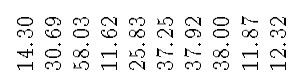 & $\stackrel{5}{50}$ \\
\hline & 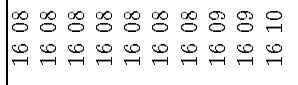 & 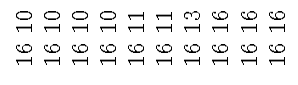 & 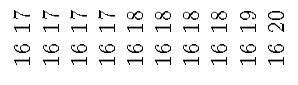 & 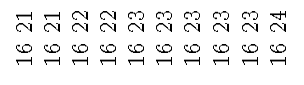 & 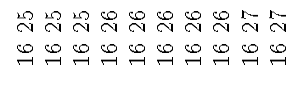 & 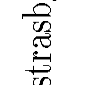 \\
\hline 急 & 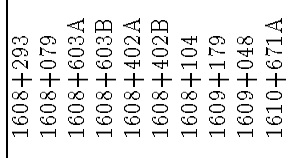 & 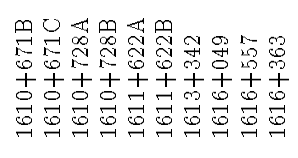 & 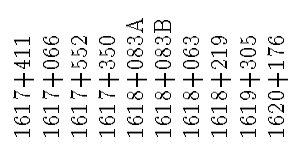 & 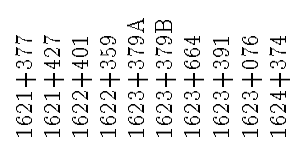 & 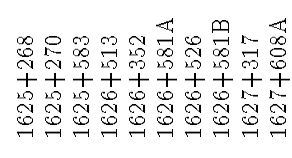 & 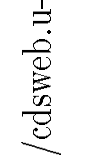 \\
\hline & & & & & & \\
\hline 20 & * $\quad * \quad *$ & * $\quad *$ & & & * & \\
\hline 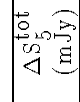 & 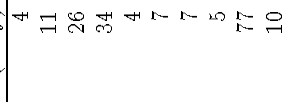 & r & $0 \underset{7}{*}$ & 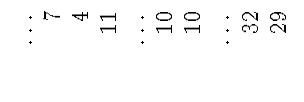 & 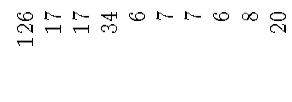 & 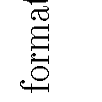 \\
\hline 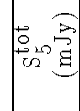 & 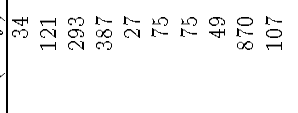 & 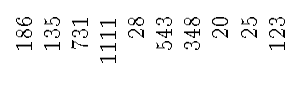 & 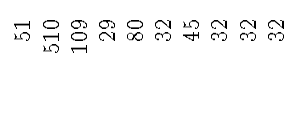 & 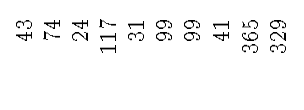 & 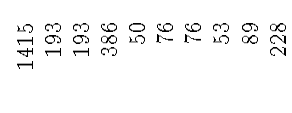 & $\frac{0}{\frac{0}{0}}$ \\
\hline 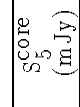 & 舟 & 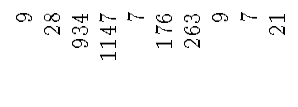 & 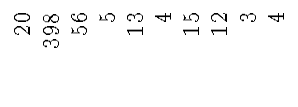 & 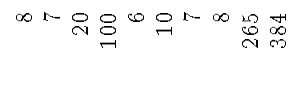 & 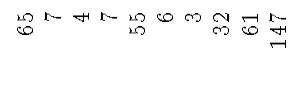 & 咅 \\
\hline$\frac{z}{z_{2}}$ & 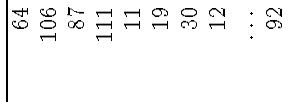 & 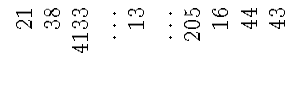 & 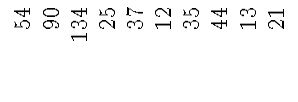 & 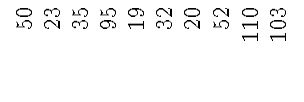 & : & $\begin{array}{l}\Xi \\
8 \\
8 \\
. \Xi\end{array}$ \\
\hline$\stackrel{2}{\circ}$ & $\operatorname{sic} 6 \operatorname{sentivis}$ & مอ & vob & 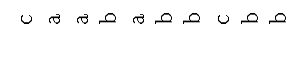 & 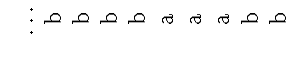 & \\
\hline$\infty$ & 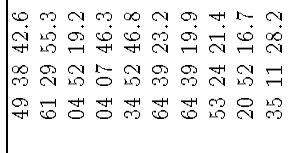 & 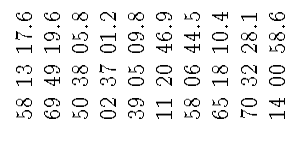 & 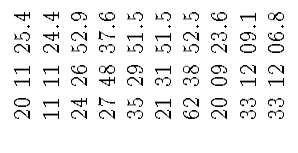 & 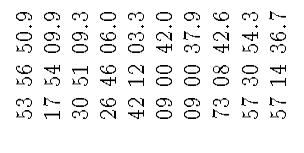 & 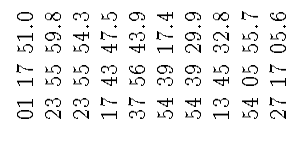 & 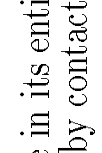 \\
\hline 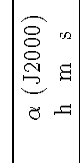 & 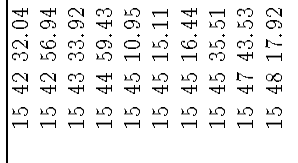 & 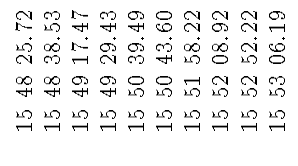 & 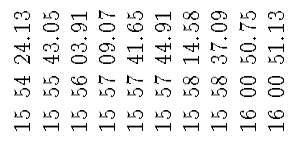 & 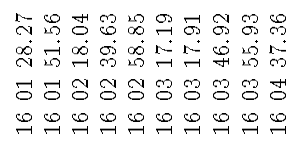 & 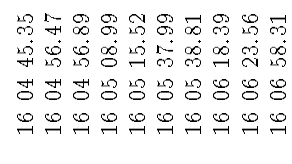 & 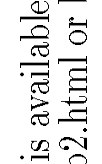 \\
\hline 莺 & 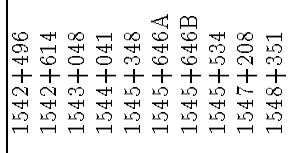 & 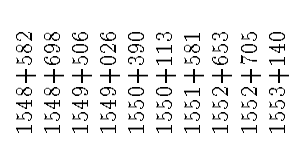 & 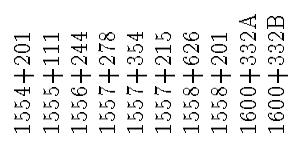 & 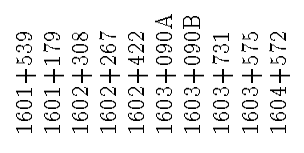 & 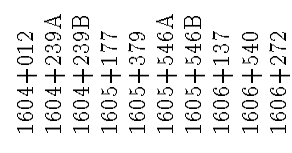 & 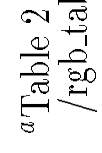 \\
\hline
\end{tabular}


Table 3. Radio source properties: low resolution data (sample page)
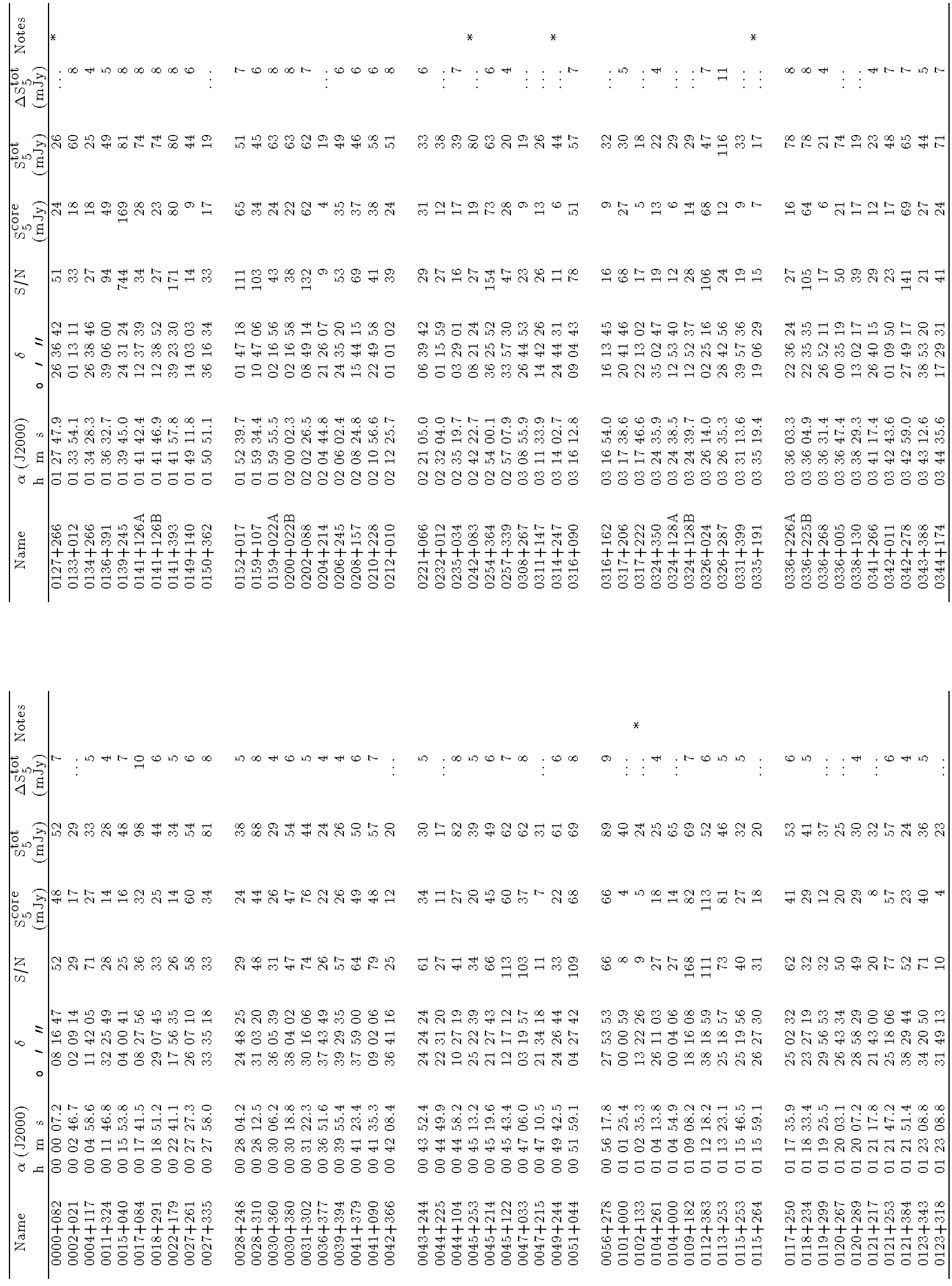

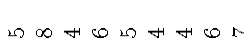

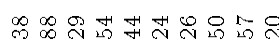

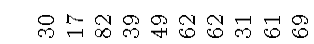

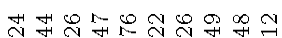

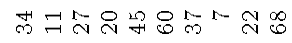

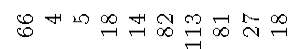

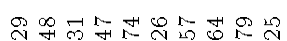

는

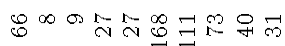

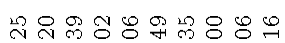

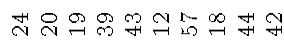

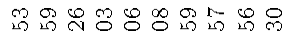

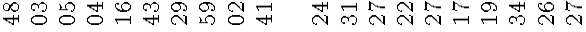

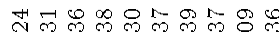

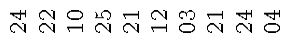

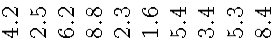

A

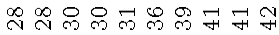

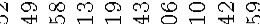
8888888888

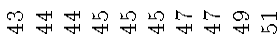
8888888888

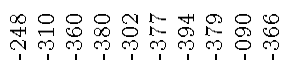

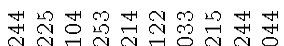

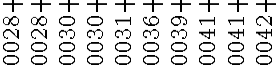

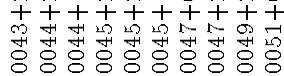
긍

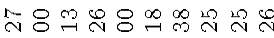

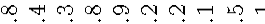

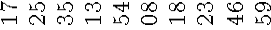

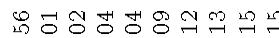
855555555

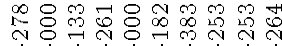

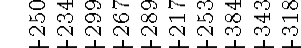

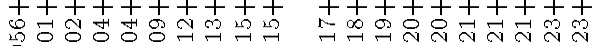

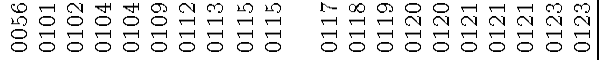

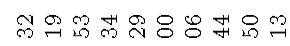

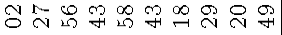

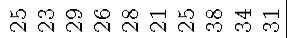

ब. म म

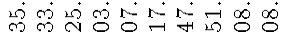

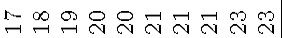
ㄷऽㄷㄷㄷㄷㅇ 
Table 4. Radio source properties: empty fields
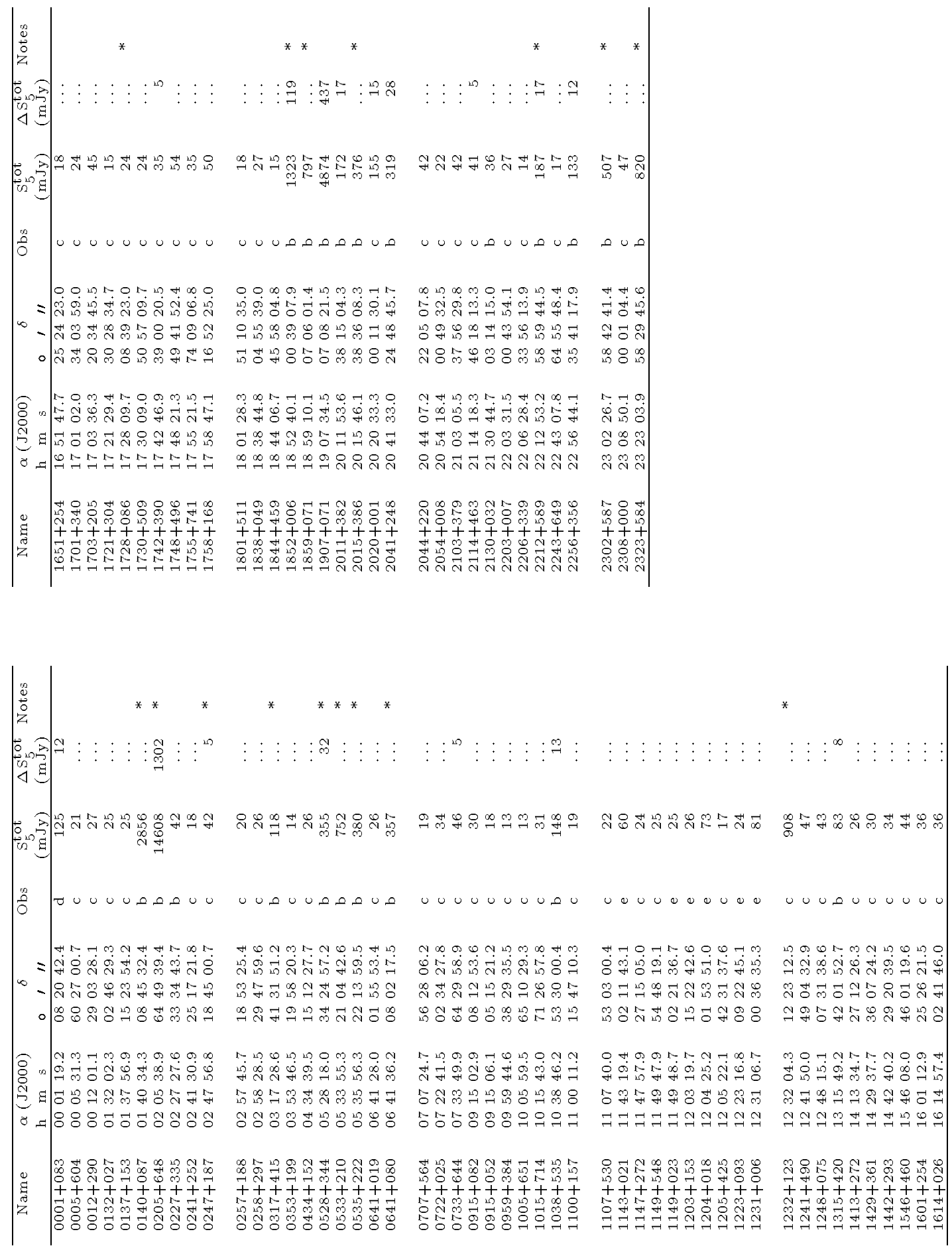